\title{
Hospital Malnutrition, Inflammation, and Cardiovascular Diseases
}

\author{
Camila Weschenfelder ${ }^{1}$ and Aline Marcadenti ${ }^{1,2}$ \\ Instituto de Cardiologia do Rio Grande do Sul/Fundação Universitária de Cardiologia, ${ }^{2}$ Rio Grande do Sul, RS - Brazil \\ Hospital do Coração (Hcor), ${ }^{2}$ São Paulo, SP - Brazil \\ Editorial related to the article: Prevalence of Malnutrition and Its Association with Clinical Complications in Hospitalized Cardiac Patients: \\ Retrospective Cohort Study
}

Malnutrition in hospitalized patients, whose prevalence ranges between $40 \%$ and $60 \%$ in Latin America, ${ }^{1}$ is related to longer hospital stays, worse clinical outcomes, ${ }^{2}$ and increased hospitalization costs. ${ }^{3}$ The consequences related to hospital malnutrition have also been reported among patients diagnosed with cardiovascular disease (CVD). ${ }^{4}$ In these individuals, an exacerbated inflammatory state is associated with malnutrition, loss of muscle mass, and cardiac cachexia, especially in the more advanced stages of the disease. ${ }^{5}$

Globally, there is a policy that aims to recognize the importance of hospital malnutrition and its consequences. ${ }^{6}$ In Brazil, in 2018, the campaign "Say no to malnutrition" was published, composed of 11 steps to combat hospital malnutrition. ${ }^{7}$ In this campaign, the first step involves risk stratification and nutritional assessment for subsequent implementation and monitoring of appropriate nutritional therapy.

In a retrospective cohort published by Ávila et al., 8 130 cardiac patients admitted to a referral hospital underwent nutritional assessment, among whom, 27\% were malnourished. In comparison to well-nourished patients, these individuals were older, needed longer time in the intensive care unit (ICU), and stayed more than 7 days in the hospital - data in accordance with the literature. ${ }^{2,4}$ In this study, the prevalence of malnutrition was lower than those reported in the literature among the general population, ${ }^{1}$ which may have occurred due to the profile of the evaluated patients, who, for the most

\section{Keywords}

Cardiovascular Diseases; Hospitalized Patients; Hospitalization; Nutrition Service Hospitalar/ administration and organization; Cachexia; Desnutrition. part, were classified as low metabolic stress. Patients using enteral and/or parenteral nutritional therapy, and patients admitted to the emergency room, ICU, or undergoing immediate surgery, were not included in the study. Thus, patients who were assessed for nutritional status may not have reflected the population with CVD with a more pronounced and/or severe inflammatory state, underestimating the frequency of malnutrition in this population.

Inflammation plays an important role at the time of nutritional diagnosis. Seeking to standardize the criteria used for the diagnosis of malnutrition, a consensus of the European Society for Enteral and Parenteral Nutrition (ESPEN $)^{9}$ proposes the use of the Global Leadership Initiative on Malnutrition (GLIM) tool. This tool includes phenotypic criteria (non-volitional weight loss, low body mass index, and reduced muscle mass) and etiologic criteria (reduced food intake or assimilation, and inflammation or disease burden). However, as this tool includes the measurement of the muscular compartment by validated body composition techniques, which may not be common in clinical practice, due to the necessary protocols or the costs involved, the GLIM tool ends up rarely being used in daily routines.

By contrast, the association between excess weight and CVD is widely recognized..$^{10}$ Patients evaluated in this study seem to reflect the population of wards in hospital cardiology units affected by ischemic diseases in milder forms and/or with a lesser degree of inflammation of the disease, for example, angina ( $25 \%$ of the sample evaluated).

Finally, malnutrition is frequent in the hospital environment and needs to be investigated early by nutritionists, especially among patients affected by CVD, whose mortality is high. Adequate nutritional diagnosis

Mailing Address: Camila Weschenfelder

Av. Princesa Isabel, 395. Postal Code: 90040-371, Porto Alegre, RS - Brazil.

E-mail: camilawesche@gmail.com 
and the establishment of adequate nutritional therapy for treatment enable the improvement of the patient's quality of life, as well as a reduction in complications and costs related to hospitalization. The article published by Ávila et al., ${ }^{8}$ demonstrates the importance of the early

\section{References}

1. Correia MI, Perman MI, Waitzberg DL. Hospital malnutrition in Latin America: A systematic review. Clin Nutr 2016; pii: S02615614(16)30160-1.

2. Ordoñez AM, Madalozzo Schieferdecker ME, Cestonaro T, Cardoso Neto J, Ligocki Campos AC. Nutritional status influences the lenght of stay and clinical outcomes in hospitalized patients in internal medicine wards. NutrHosp 2013; 28(4):1313-1320.

3. Curtis LJ, Bernier P, Jeejeebhoy K, Allard J, Duerksen D, Gramlich L, et al. Costs of hospital malnutrition. Clin Nutr 2017;36(5):13911396.

4. Ruiz AJ, Buitrago G, Rodríguez N, Gómez G, Sulo S, Gómez C, et al. Clinical and economic outcomes associated with malnutrition in hospitalized patients. Clin Nutr.2019;38(3):1310-6.

5. Rahman A, Jafry S, Jeejeebhoy K, Nagpal AD, Pisani B, Agarwala R. Malnutrition and Cachexia in Heart Failure. JPEN J Parenter Enteral Nutr .2016;40(4):475-86. identification of the nutritional status of hospitalized patients with CVD, for immediate implementation of appropriate nutritional therapy aimed at reducing hospital malnutrition and, therefore, the reduction of associated outcomes, such as ICU and hospital stay.

6. Kirkland LL, Shaughnessy E. Recognition and Prevention of Nosocomial Malnutrition: A Review and A Call to Action! Am J Med. 2017;130(12):1345-50.

7. Toledo DO, Piovacari SMF, Horie LM, Nunes L, Castro MG, Ceniccola GD, et al. Campanha "Diga não à desnutrição": 11 passos importantes para combater a desnutrição hospitalar. BRASPEN J 2018; 33: 86-100.

8. Ávila NG, Carneiro JU, Alves FD, Corrêa IVS, Vallandro JP. Prevalence of Malnutrition and Its Association with Clinical Complications in Hospitalized Cardiac Patients: Retrospective Cohort Study. Int J Cardiovasc Sci.2020; 33(6):629-634.

9. Jensen GL, Cederholm T, Correia MITD, Gonzalez MC, Fukushima R, Higashiguchi T, et al. GLIM Criteria for the Diagnosis of Malnutrition: A Consensus Report From the Global Clinical Nutrition Community. JPEN J Parenter Enteral Nutr. 2019;43(1):32-40.

10. Neeland IJ, Poirier P, Després JP. Cardiovascular and Metabolic Heterogeneity of Obesity: Clinical Challenges and Implications for Management. Circulation 2018;137(13):1391-406. 